\title{
On the permutation groups of cyclic codes
}

\author{
Kenza Guenda • T. Aaron Gulliver
}

Received: 20 November 2011 / Accepted: 11 September 2012 / Published online: 3 October 2012

(C) Springer Science+Business Media, LLC 2012

\begin{abstract}
We classify the permutation groups of cyclic codes over a finite field. As a special case, we find the permutation groups of non-primitive $\mathrm{BCH}$ codes of prime length. In addition, the Sylow $p$-subgroup of the permutation group is given for many cyclic codes of length $p^{m}$. Several examples are given to illustrate the results.
\end{abstract}

Keywords Permutation groups · Transitive groups · Doubly transitive groups · Non-primitive $\mathrm{BCH}$ codes

\section{Introduction}

The permutation groups of cyclic codes are of great theoretical and practical interest, e.g. the permutation group can be used to find the weight distribution of a code [19], and in decoding $[16,19]$. They can also be used for cryptographic purposes such as the McEliece cryptosystem and its variants [20]. Despite the significance of this problem, the permutation groups of cyclic codes are known for only a few subclasses such as the Reed-Solomon codes, Reed-Muller codes and some BCH codes [3, 18]. The other cases remain open. Recently, Bienert and Klopsch [4] studied the permutation groups of cyclic codes in the binary case. They gave the primitive groups which can be the permutation group of a binary cyclic code. Dobson and Witte [13, 14] considered the cyclic codes invariant under some transitive groups. Furthermore in some cases they gave the Sylow $p$-subgroups of some transitive subgroups of $S_{p^{2}}$ and $S_{p^{m}}$.

K. Guenda

Faculty of Mathematics USTHB, University of Science and Technology of Algiers, Algiers, Algeria

T. Aaron Gulliver $(\bowtie)$

Department of Electrical and Computer Engineering, University of Victoria, PO Box 3055, STN

CSC, Victoria, BC, V8W 3P6, Canada

e-mail: agullive@ece.uvic.ca 
In this paper we classify the permutation groups of cyclic codes. First we generalize the results of [4] concerning the doubly transitive permutation groups with socle $\operatorname{PSL}(d, q)$ to the non-binary case. Then we use the classification of the doubly transitive groups which contain a complete cycle, given by McSorley [21, 22], and our previous results to determine the permutation groups in the doubly transitive cases. This allows us to determine the permutation groups of the $\mathrm{BCH}$ codes in the prime length case. Further, we give conditions on the primitivity of the permutation groups which are based on the underlying field and the length of the code. For many cyclic codes, we explicitly give the Sylow $p$-subgroups of the permutation groups in the primitive and imprimitive cases. This is done using some subgroups of $S_{p^{m}}$ introduced by Brand [5]. Several examples are given to illustrate the results.

\section{Preliminaries}

Let $\mathbb{F}_{q}$ be a finite field. A linear code $C$ of length $n$ over $\mathbb{F}_{q}$ is a subspace of $\mathbb{F}_{q}^{n}$. A vector $x=\left(x_{0}, \ldots, x_{n-1}\right) \in C$ is said to be a codeword of $C$. Let $I$ denote the set $\{0,1, \ldots, n-1\}$, and let $S_{n}$ be the symmetric group acting on $I$. Then $S_{n}$ acts naturally on a codeword of $C$ as follows. If $\sigma$ is a permutation of $S_{n}$, then

$$
\sigma(x)=\left(x_{\sigma^{-1}(0)}, \ldots, x_{\sigma^{-1}(n-1)}\right), \quad\left(x_{0}, \ldots, x_{n-1}\right) \in C .
$$

The permutation group of $C$ is the subgroup of $S_{n}$ given by

$$
\operatorname{Per}(C)=\left\{\sigma \in S_{n} \mid \sigma(C)=C\right\} .
$$

A linear code $C$ over $\mathbb{F}_{q}$ is cyclic if $T \in \operatorname{Per}(C)$, where $T=(0,1, \ldots, n-1)$ is a complete cycle of length $n$. If $C$ is cyclic then $\operatorname{Per}(C)$ is a transitive group. The group $A G(n)=\left\{\tau_{a, b}: a \neq 0,(a, n)=1, b \in \mathbb{Z}_{n}\right\}$ is the subgroup of $S_{n}$ formed by the permutation defined as follows:

$$
\begin{aligned}
\tau_{a, b}: \mathbb{Z}_{n} & \longrightarrow \mathbb{Z}_{n} \\
x & \longmapsto(a x+b) \bmod n .
\end{aligned}
$$

The group $A G(n)$ is called the group of affine transformations. The affine transformations $M_{a}=\tau_{a, 0}$ are called a multiplier. The affine group $A G L(1, p)$ is the group of affine transformations over $\mathbb{Z}_{p}$. The projective semi-linear group $P \Gamma L(d, t)$ is the semi-direct product of the projective linear group $P G L(d, t)$ and the automorphism group $Z=\operatorname{Gal}\left(\mathbb{F}_{t} / \mathbb{F}_{p}\right)$ of $\mathbb{F}_{t}$, where $t=p^{s}, p$ prime, i.e.

$$
P \Gamma L(d, t)=P G L(d, t) \rtimes Z \text {. }
$$

Remark 1 The zero code, the entire space, and the repetition code and its dual are called elementary codes. The permutation group of these codes is $S_{n}$ [16, p. 1410]. Further, it was proven in [16, p. 1410] that there is no cyclic code with permutation group equal to $\operatorname{Alt}(n)$. 


\section{The permutation groups of cyclic codes}

A doubly transitive group $G$ has a unique minimal normal subgroup $N$ which is either regular and elementary abelian, or simple and primitive, and the centralizer of $N$ in $G$ is equal to $C_{G}(N)=1[8$, p. 202]. All simple groups which can occur as a minimal normal subgroup of a doubly transitive group are known. This result is due to the classification of finite simple groups [9]. Using this classification, McSorley [21] gave the following result.

Lemma 1 A group $G$ of degree $n$ which is doubly transitive and contains a complete cycle has socle $N$ with $N \leq G \leq A u t(N)$, and is equal to one of the cases in Table 1 .

The arguments given in the following Lemma are similar to those for the binary case [4, Theorem E, Part 3].

Lemma 2 Let $C$ be a non-elementary cyclic code of length $n=\frac{t^{d}-1}{t-1}$ over a finite field $\mathbb{F}_{q}$, where $q=r^{\alpha}$ and $t$ is a prime power. If the group $\operatorname{Per}(C)$ satisfies

$$
P G L(d, t) \leq \operatorname{Per}(C) \leq P \Gamma L(d, t),
$$

then $t=r^{a}$ for some $a \geq 1, d \geq 3$, and $\operatorname{Per}(C)=P \Gamma L(d, t)$.

Proof Assume $d=2$. As the group $P G L(2, t)$ acts 3-transitively on the 1dimensional projective space $\mathbb{P}^{1}\left(\mathbb{F}_{t}\right)$, we deduce from [22, Table 1 and Lemma 2], that the underlying code is elementary, which is a contradiction. Hence $d \geq 3$, and from [22, Table 1 and Lemma 2], it must be that since $C$ is non-elementary, $t$ must be equal to $r^{a}$. Now let $V$ denote the permutation module over $\mathbb{F}_{r}$ associated with the natural action of $P G L(d, t)$ on the $(d-1)$-dimensional projective space $\mathbb{P}^{d-1}\left(\mathbb{F}_{t}\right)$. Let $U_{1}$ be a $P G L(d, t)$-submodule of $V$. Then $U_{1}$ is $P \Gamma L(d, t)$-invariant. This is because, if $\sigma$ is a generator of the cyclic group $P \Gamma L(d, t) / P G L(d, t) \simeq \operatorname{Gal}\left(\mathbb{F}_{t} / \mathbb{F}_{r}\right)$, then $U_{2}=U_{1}^{\sigma}$, regarded as a $P G L(d, t)$-module, is simply a twist of $U_{1}$. Let $\overline{\mathbb{F}}_{r}$

Table 1 The doubly transitive groups that contain a complete cycle

\begin{tabular}{lll}
\hline$G$ & $n$ & $N$ \\
\hline$A G L(1, p)$ & $p$ & $C_{p}$ \\
$S_{4}$ & 4 & $C_{2} \times C_{2}$ \\
$S_{n}, n \geq 5$ & $n$ & Alt $(n)$ \\
Alt $(n), n$ odd and $\geq 5$ & $n$ & Alt $(n)$ \\
$P G L(d, t) \leq G \leq P \Gamma L(d, t)$ & $\frac{t^{d}-1}{t-1}$ & $P S L(d, t)$ \\
$(d, t) \neq(2,2),(2,3),(2,4)$ & & \\
$P S L(2,11)$ & 11 & $P S L(2,11)$ \\
$M_{11}($ Mathieu $)$ & 11 & $M_{11}($ Mathieu $)$ \\
$M_{23}$ (Mathieu) & 23 & $M_{23}($ Mathieu $)$ \\
\hline
\end{tabular}


be the algebraic closure of $\mathbb{F}_{r}$. Then the composition factors of the $\overline{\mathbb{F}}_{r} P G L(d, t)$ modules $\bar{U}_{1}=\overline{\mathbb{F}}_{r} \otimes U_{1}$ and $\bar{U}_{2}=\overline{\mathbb{F}}_{r} \otimes U_{2}$ are the same. The submodules of the $\overline{\mathbb{F}}_{r} P G L(d, t)$-module $\bar{V}=\overline{\mathbb{F}}_{r} \otimes V$ are uniquely determined by their composition factors [1]. Then we have $\bar{U}_{1}=\bar{U}_{2}$, which implies that $U_{1}=U_{2}$, and therefore $\operatorname{Per}(C)=P \Gamma L(d, t)$

The following theorem establishes the permutation group of a non-elementary cyclic code of prime length over $\mathbb{F}_{q}$, where $q=r^{\alpha}$.

Theorem 3 Let $C$ be a non-elementary cyclic code of length $p$ over $\mathbb{F}_{q}$. Then $\operatorname{Per}(C)$ is a primitive group, and one of the following holds:

(i) $\operatorname{Per}(C)$ is a solvable group of order pm with $m$ a divisor of $p-1$ and $C_{p} \leq$ $\operatorname{Per}(C) \leq A G L(1, p)$, with $p \geq 5$. Furthermore Per $(C)$ contains a normal Sylow p-subgroup.

(ii) If $p=q$, then $\operatorname{Per}(C)=A G L(1, p)$.

(iii) $\operatorname{Per}(C)=P S L(2,11)$ and $q$ is a power of 3. $C$ is either the $[11,6]$ or $[11,5]$ code that is equivalent to the $[11,6,5]$ ternary Golay code or its dual, respectively.

(iv) $\operatorname{Per}(C)=M_{23}$ and $q$ is a power of $2 . C$ is either the $[23,12]$ or $[23,11]$ code that is equivalent to the $[23,12,7]$ binary Golay code or its dual, respectively.

(v) $\operatorname{Per}(C)=P \Gamma L\left(d, r^{d^{b}}\right)$ where $b \in \mathbb{N}, d \geq 3$ is a prime number such that $\left(d, r^{d^{b}}-1\right)=1$, and $p=\left(r^{d^{b+1}}-1\right) /\left(r^{d^{b}}-1\right)$.

Proof A transitive group of prime degree is a primitive group [23, p. 195]. As a consequence of a result of Burnside [13, Theorem 2], a transitive group of prime degree is either a subgroup of $A G L(1, p)$ or a doubly transitive group. In the first case $C_{p} \leq \operatorname{Per}(C) \leq A G L(1, p)$, and if $p=2$ or $3, A G L(1, p)=S_{p}$. In this case, $C$ is elementary by Remark 1 , which is a contradiction. Since $C_{p}$ is normal in $A G L(1, p)$ and $A G L(1, p) / C_{p}$ is abelian, $\operatorname{Per}(C)$ is a normal subgroup. By [11, Example 3.5.1] $G$ is solvable. If $q=p$, Roth and Seroussi [24] proved that any cyclic code of prime length $p$ over $\mathbb{F}_{p}$ must be an MDS code equivalent to an extended ReedSolomon code. Berger [2] proved that the permutation group of such codes is the affine group $A G L(1, p)$. In the doubly transitive cases, as $C$ is non-elementary of prime length $p$, by Lemma 1, Remark 1 and Lemma 2, we see that $\operatorname{Per}(C)$ is one of $M_{11}$, with $p=11, P S L(2,11)$ with $p=11, M_{23}$ with $p=23$, or $P \Gamma L(d, t)$ of degree $p=\left(t^{d}-1\right) /(t-1)$ and $t$ a prime power. If $\operatorname{Per}(C)=M_{11}$, from [22, Table 1, Lemma 2] $C$ must be elementary, which is a contradiction. If $\operatorname{Per}(C)=P S L(2,11)$, from [22, Table 1, Lemma 2 and (J)] $q$ must be a power of 3 , and there is a unique nonelementary code over $\mathbb{F}_{q}$ contained in the dual of the repetition code. The $[11,5,6]$ dual of the ternary Golay code is contained in the repetition code and has permutation group PSL $(2,11)$; its dual, an $[11,6,5]$ code, intersects the dual of the repetition code in this $[11,5,6]$ code and also has permutation group $P S L(2,11)$. Part (ii) then follows. Part (iii) is obtained in an analogous way from [22, Table 1, Lemma 2 and (I)]. For Part (iv), we have from Lemma 2 that $\operatorname{Per}(C)=P \Gamma L(d, t), t=r^{a}$ for some $a \geq 1$ and $d \geq 3$. A number theory argument [12, Lemma 3.1] gives the result 
Table 2 Permutation groups of some BCH codes of length $p$

\begin{tabular}{llllll}
\hline$q$ & $p$ & $\delta$ & $\operatorname{Per}(C)$ & $\operatorname{Per}\left(C_{2}\right)$ & $\operatorname{Per}\left(C_{3}\right)$ \\
\hline 2 & 17 & 2 & $C_{8} \ltimes C_{17}$ & $S_{17}$ & $S_{17}$ \\
2 & 23 & 3 & $M_{23}$ & $M_{23}$ & $M_{23}$ \\
2 & 41 & 2 & $C_{20} \ltimes C_{41}$ & $C_{20} \ltimes C_{41}$ & $C_{20} \ltimes C_{41}$ \\
2 & 41 & 3 & $C_{20} \ltimes C_{41}$ & $S_{41}$ & $S_{41}$ \\
2 & 43 & 5 & $C_{14} \ltimes C_{43}$ & $C_{14} \ltimes C_{43}$ & $C_{14} \ltimes C_{43}$ \\
2 & 43 & 7 & $C_{14} \ltimes C_{43}$ & $S_{43}$ & $S_{43}$ \\
3 & 13 & 2 & $C_{3} \ltimes C_{13}$ & $C_{3} \ltimes C_{13}$ & $C_{3} \ltimes C_{13}$ \\
3 & 13 & 4 & $P \Gamma L(3,3)$ & $C_{3} \ltimes C_{13}$ & $C_{3} \ltimes C_{13}$ \\
3 & 13 & 5 & $C_{3} \ltimes C_{13}$ & $C_{3} \ltimes C_{13}$ & $C_{3} \ltimes C_{13}$ \\
3 & 23 & 3 & $C_{11} \ltimes C_{23}$ & $C_{11} \ltimes C_{23}$ & $C_{11} \ltimes C_{23}$ \\
3 & 41 & 5 & $C_{8} \ltimes C_{41}$ & $C_{8} \ltimes C_{41}$ & $C_{8} \ltimes C_{41}$ \\
4 & 43 & 9 & $C_{7} \ltimes C_{43}$ & $S_{43}$ & $S_{43}$ \\
5 & 11 & 5 & $C_{5} \ltimes C_{11}$ & $C_{5} \ltimes C_{11}$ & $C_{5} \ltimes C_{11}$ \\
11 & 5 & 3 & $C_{5}$ & $C_{2} \ltimes C_{5}$ & $C_{5}$ \\
\hline
\end{tabular}

that if $p$ is prime, then $d$ must be a prime such that $\left(d, r^{a}-1\right)=1$ and $a=b^{d}$. The result then follows.

Remark 2 For $p$ prime, the permutation group of a non-elementary $\mathrm{BCH}$ code of length $p$ over $\mathbb{F}_{q}$ is one of those listed in Theorem 3 .

In Table 2, we give examples of permutation groups of $\mathrm{BCH}$ codes of length $p$ over $\mathbb{F}_{q} . \operatorname{Per}(C)$ (respectively $\operatorname{Per}\left(C_{2}\right)$ and $\operatorname{Per}\left(C_{3}\right)$ ), denotes the permutation group of the narrow sense $(b=1) \mathrm{BCH}$ code with designed distance $\delta$ (respectively $\mathrm{BCH}$ code with designed distance $\delta$ and $b=2$ and $b=3$ ).

The following result is obtained by considering the permutation groups of cyclic codes of composite length.

Theorem 4 Let $C$ be a non-elementary cyclic code over $\mathbb{F}_{r^{\alpha}}$ of composite length. Then $\operatorname{Per}(C)$ is either

(i) an imprimitive group (in the case that $n=p^{m}, p$ a prime, the orbit of the subgroup generated by $T^{p^{m-1}}$ and its conjugate form a complete block system of $\operatorname{Per}(C))$ or

(ii) $\operatorname{Per}(C)$ is a doubly transitive group equal to

$$
P \Gamma L\left(d, r^{a}\right), \quad \text { with } n=\frac{r^{a d}-1}{r^{a}-1}, d \geq 3, a \geq 1 .
$$

Proof The group $\operatorname{Per}(C)$ contains a complete cycle and has composite degree. Hence from a theorem of Burnside and Schur [25, p. 65], $\operatorname{Per}(C)$ is either imprimitive or doubly transitive. If it is imprimitive and $n=p^{m}$, by [7, Chap. XVI, Theo- 
rem VIII] $\operatorname{Per}(C)$ contains an intransitive normal subgroup generated by $T^{p^{m-1}}$ and its conjugates. By [25, Proposition 7.1] the orbit of such a subgroup forms a complete block system of $\operatorname{Per}(C)$.

In the doubly transitive case, we have from Lemma 1 that the only cases when the socle can be abelian are $N=C_{p}$ and $N=C_{2} \times C_{2}$. In these cases, $\operatorname{Per}(C)$ must be equal to $A G L(1, p)$ or $S_{4}$, which is impossible. Since the socle is not abelian and the degree is not prime, this leads to the only solution given by row six of Table 1 in Lemma 1. Hence from Lemma 2, Part (ii) follows.

\section{The permutation group of cyclic codes of prime power length}

In this section, we consider the permutation groups of cyclic codes of length $p^{m}$, where $p$ is an odd prime.

Lemma 5 Let $q$ be a prime power, $p$ an odd prime, and $z$ the largest integer such that $p^{z} \mid\left(q^{t}-1\right)$, with $t$ the order of $q$ modulo $p$. If $z=1$ we have

$$
\operatorname{ord}_{p^{m}}(q)=p^{m-1} t \text {. }
$$

Proof Let $t$ be the order of $q$ modulo $p$, and $u=q^{t} \equiv 1 \bmod p$. Assume that $z=1$, or equivalently $u \neq 1 \bmod p^{2}$. It is well known from elementary number theory [10, p. 87] that $u \bmod p^{m}$ is an element of order $p^{m-1}$ in the group $\left(\mathbb{Z}_{p^{m}}\right)^{*}$ if and only if $u \neq 1 \bmod p^{2}$. Hence $\operatorname{ord}_{p^{m}}(q)=p^{m-1} t$.

According to Brillhart et al. [6], it is unusual to have $z>1$.

Proposition 6 Let $n=p^{m}$ and $q=r^{\alpha}$ a prime power with $(q, n)=1$, and $C$ a cyclic code of length $n$ over $\mathbb{F}_{q}$. Let $M_{q}$ be the multiplier defined by $M_{q}(i)=i q \bmod p^{m}$. Then the group Per $(C)$ contains the subgroup $K=\left\langle T, M_{q}\right\rangle$ of order $p^{m} \operatorname{ord}_{p^{m}}(q)$. Let $p^{l}$, with $l \geq m$ be the p-part of the order of $K$. Then a Sylow $p$-subgroup $P$ of $\operatorname{Per}(C)$ has order $p^{s}$ such that

$$
l \leq s \leq p^{m-1}+p^{m-2}+\cdots+1 .
$$

If $z=1$, then $s \geq 2 m-1$.

Proof By the definition of a cyclic code, we have $T \in \operatorname{Per}(C)$. It is obvious that each cyclotomic class modulo $n$ over $\mathbb{F}_{q}$ is invariant under the permutation $M_{q}$. This can be deduced from the fact that the polynomial $f(x) \in \mathbb{F}_{q}[x]$ satisfies $f\left(x^{q}\right)=f(x)^{q}$. Thus $M_{q} \in \operatorname{Per}(C)$. The order of $M_{q}$ is equal to $\operatorname{ord}_{n}(q)$, hence $K=\left\langle T, M_{q}\right\rangle$ is a subgroup of $\operatorname{Per}(C)$ of order $n \operatorname{ord}_{n}(q)$. Since $n=p^{m}$, the order of $K$ has $p$ part $p^{l}$ with $l \leq m$. Let $P$ be a Sylow $p$-subgroup of $\operatorname{Per}(C)$ which contains $T$. Then $P$ is a $p$-subgroup of $S_{p^{m}}$. From Sylow's Theorem, $P$ is contained in a Sylow $p$-subgroup of $S_{p^{m}}$. It is well known that a Sylow $p$-subgroup of $S_{p^{m}}$ is of order $p^{p^{m-1}+p^{m-2}+\cdots+1}[23$, Kalužnin's Theorem]. Since $P$ also contains the subgroup of $K$ of order $p^{l}$, then $l \leq s \leq p^{m-1}+p^{m-2}+\cdots+1$. If $z=1$, then by Lemma 5 the 
order of the group $K$ is $\operatorname{ord}_{p}(q) p^{2 m-1}$. This shows that $p^{2 m-1}$ divides $|\operatorname{Per}(C)|$, so $\operatorname{Per}(C)$ contains a $p$-subgroup of order at least $p^{2 m-1}$.

Theorem 7 Let $C$ be a non-elementary cyclic code of length $p^{m}$ over $\mathbb{F}_{r^{\alpha}}$, with $m \geq 1$. Then the following hold:

(i) If $p \nmid \alpha$ and $p \nmid\left(d, r^{a}-1\right)$, then $\operatorname{Per}(C)=P \Gamma L\left(d, r^{a}\right), a \geq 1, d \geq 3$, if and only if the Sylow p-subgroup of $\operatorname{Per}(C)$ is of order $p^{m}$.

(ii) If $p \geq 5, \alpha=1$ and $r=p, m>1$, then $\operatorname{Per}(C)$ is an imprimitive group which admits a complete system formed by the orbit of the subgroup generated by $T^{p^{m-1}}$ and its conjugate. It also contains a transitive normal Sylow p-subgroup of order $p^{s}$ with $m<s \leq p^{m-1}+p^{m-2}+\cdots+1$.

(iii) If $z=1, p \nmid \alpha$ and $p \nmid\left(d, r^{a}-1\right)$, then $\operatorname{Per}(C)$ is an imprimitive group which contains a transitive normal Sylow p-subgroup of order $p^{s}$, with $2 m-1 \leq s \leq$ $p^{m-1}+p^{m-2}+\cdots+1$. Furthermore, $\operatorname{Per}(C)$ admits a complete block system formed by the orbit of the subgroup generated by $T^{p^{m-1}}$ and its conjugate.

Proof For Part (i), we know that the socle of $P \Gamma L\left(d, r^{a}\right)$ is the group $P S L\left(d, r^{a}\right)$ of order $\frac{r^{a d(d-1) / 2}}{\left(d, r^{a}-1\right)} \prod_{i=2}^{d}\left(r^{a i}-1\right)$. From a lemma of Zsigmondy [17, Chap. IX, Theorem 8.3], except for the cases $d=2, r^{a}=2^{b}-1$ and $d=6, r^{a}=2$, there exists a prime $q_{0}$ such that $q_{0}$ divides $r^{a d}-1$, but does not divide $r^{a i}-1$, for $1 \leq i<d$. From Lemma 2, we cannot have $d=2$. The case $d=6$ and $r^{a}=2$ does not give a prime power. Hence if $n=p^{m}=\frac{r^{a d}-1}{r^{a}-1}$, there is a $q_{0}$ which divides $\left(r^{a d}-1\right)=\left(r^{a}-1\right) p^{m}$. Since $q_{0}$ does not divide $r^{a}-1$, then $q_{0}$ divides $p^{m}$, and hence $q_{0}=p$ and $p^{m}$ is the $p$-part of the order of $P S L\left(d, r^{a}\right)$. Also, since $p \nmid r^{a}-1$, we have $p \nmid\left(d, r^{a}-1\right)$. Hence if $(\alpha, p)=1, p^{m}$ is also in the $p$-part of the order of $P \Gamma L\left(d, r^{a}\right)$, and the result follows.

Conversely, if $\operatorname{Per}(C)$ has Sylow $p$-subgroup $P$ of order $p^{m}$, we can assume that $T \in P$, which gives the equality $P=\langle T\rangle$. Assume that in this case $\operatorname{Per}(C)$ is imprimitive. Then by [13, Theorem 33], $P$ is normal. $P$ is then the minimal normal subgroup which is transitive and abelian. From [25, p. 17] $\operatorname{Per}(C)$ is primitive, which is impossible. Thus if $P=\langle T\rangle$, the group $\operatorname{Per}(C)$ is equal to $P \Gamma L\left(d, r^{a}\right)$, which is possible only if $\left[P \Gamma L\left(d, r^{a}\right): P S L\left(d, r^{a}\right)\right]$ is prime to $p$, i.e, $(p, \alpha)=1$ and $p \nmid\left(d, r^{a}-1\right)$.

For Part (ii), from Theorem 4 if $\operatorname{Per}(C)$ is primitive, then it is doubly transitive and equal to $P \Gamma L\left(d, r^{a}\right)$ with $n=\frac{r^{a d}-1}{r^{a}-1}, d \geq 3$ and $a \geq 1$. From [13, Lemma 22], if $\operatorname{Per}(C)$ is doubly transitive with non abelian socle, then $\operatorname{Soc}(\operatorname{Per}(C))=\operatorname{Alt}\left(p^{m}\right)$. Hence from Remark 1 the code is elementary, which is a contradiction. Therefore, $\operatorname{Per}(C)$ is imprimitive, and then by Part (i) the order of the Sylow $p$-subgroup is $p^{s}$ with $s>m$. The second inequality then follows by Proposition 6 .

For Part (iii), if $z=1$ then from Proposition 6, we find that the order of a Sylow $p$ subgroup of $\operatorname{Per}(C)$ is at least $p^{2 m-1}$. If $\operatorname{Per}(C)$ is doubly transitive, by Theorem 4 it is equal to $P \Gamma L\left(d, r^{a}\right)$, with $d \geq 3$. By assuming $p \nmid \alpha$ and $p \nmid\left(d, r^{a}-1\right)$, we obtain from Part (i) that a Sylow $p$-subgroup of $\operatorname{Per}(C)$ has order $p^{m}$, which is impossible. Hence $\operatorname{Per}(C)$ is an imprimitive group. From [13, Theorem 33] $\operatorname{Per}(C)$ contains a transitive normal Sylow $p$-subgroup, hence the result follows. 
Example 1 The narrow sense $\mathrm{BCH}$ code of length 25 over $\mathbb{F}_{3}$ with designed distance 3 has a permutation group which is the imprimitive group $S_{5} 2 S_{5}$. The narrow sense $\mathrm{BCH}$ code of length 9 over $\mathbb{F}_{5}$ with designed distance 2 has a permutation group which is the imprimitive group $S_{3} 2 S_{3}$. The binary [7, 4, 3] Hamming code has permutation group $P \Gamma L(3,2)$, which contains a Sylow 7-subgroup of order 7 .

We now give the Sylow $p$-group of $\operatorname{Per}(C)$ for several cases. Let $p$ be an odd prime. For $n<p-1$, we define the following subsets of $S_{p^{m}}$ :

$Q^{n}=\left\{f: \mathbb{Z}_{p^{m}} \rightarrow \mathbb{Z}_{p^{m}} \mid f(x)=\sum_{i=0}^{n} a_{i} x^{i}, a_{i} \in \mathbb{Z}_{p^{m}}\right.$ for each $i, \quad\left(p, a_{1}\right)=1$, and $p^{m-1}$ divides $a_{i}$ for $\left.i=2,3, \ldots, n\right\}$.

$Q_{1}^{n}=\left\{f \in Q^{n} \mid f(x)=\sum_{i=0}^{n} a_{i} x^{i}\right.$, with $\left.a_{1} \equiv 1 \bmod p^{m-1}\right\}$.

The sets $Q^{n}$ and $Q_{1}^{n}$ are subgroups of $S_{p^{m}}$ [5, Lemma 2.1]. Note that $Q^{1}=$ $A G\left(p^{m}\right)$.

The following lemma will be used later. Note that the proof is similar to that of [15, Lemmas 2.4, 2.5].

Lemma 8 If $1 \leq n<p-1$, then

(i) $\left|Q^{n}\right|=(p-1) p^{2 m+n-2}$ and $\left|Q_{1}^{n}\right|=p^{m+n}$.

(ii) $A G\left(p^{m}\right)=N_{S_{p^{m}}}(\langle T\rangle)$.

(iii) $N_{S_{p^{m}}}\left(Q_{1}^{n}\right)=Q^{n+1}$.

Proof For Part (i), by [5, Lemma 3.2], the map $\left(a_{0}, \ldots, a_{n}\right) \longrightarrow f$, where $f(x)=$ $\sum_{i=0}^{n} a_{i} x^{i}$ is injective if $n<p-1$. Thus in $Q^{n}$, the coefficients of $a_{0}$ can take $p^{r}$ different values, and $a_{1}$ can take $p^{m-1}(p-1)$ values. For $2 \leq i \leq n, a_{i}$ can take $p$ values. From these results we have $\left|Q^{n}\right|=p^{2 m+n-2}(p-1)$. For $Q_{1}^{n}$, the coefficients of $a_{0}$ can take $p^{m}$ different values, and $a_{i}$ for $1 \leq i \leq n$ can take $p$ values, hence $\left|Q_{1}^{n}\right|=p^{m+n}$.

Now we prove that $A G\left(p^{m}\right)=N_{S_{p^{m}}}(\langle T\rangle)$. Let $\sigma$ be an element of $N_{S_{p^{m}}}(\langle T\rangle)$. Then, there is a $j \in \mathbb{Z}_{n} \backslash\{0\}$ such that $\sigma T \sigma^{-1}=T^{j}$, or equivalently $\sigma T=T^{j} \sigma$. Hence $\sigma T(0)=\sigma(1)=T^{j} \sigma(0)=\sigma(0)+j$ and $\sigma T(1)=\sigma(1)+j=\sigma(0)+2 j$. Therefore $\sigma(k)=\sigma(0)+k j$ for any $k \in \mathbb{Z}_{n}$. Then $(j, n)=1$ follows from the fact that the order of $T$ equals the order of $T^{j}$.

Now we prove Part (iii).

( $\subseteq$ part) Let $h \in N_{p^{m}}\left(Q_{1}^{n}\right)$ and $g=h^{-1} T h$. As $T \in Q_{1}^{n}$, it must be that $g \in Q_{1}^{n}$. Since the order of $g$ is equal to the order of $T$ (which is $p^{m}$ ), from [5, Lemma 3.6] there exists $f \in Q^{n+1}$ such that $f^{-1} g f=T$. Thus $f^{-1} h^{-1} T h f=T$. The only elements of $S_{p^{m}}$ which commute with $T$ (a complete cycle of length $p^{m}$ ), are the powers of $T$. Thus $h f=T^{j}$ for some $j$. Since $Q^{n+1}$ is a subgroup of $S_{p^{m}}$ and $\langle T\rangle \leq Q^{n+1}$, then $h \in Q^{n+1}$. Hence $N_{p^{m}}\left(Q_{1}^{n}\right) \leq Q^{n+1}$.

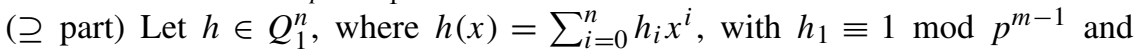
$p^{m-1} \mid h_{i}$, for $2 \leq i \leq n$. Let $g \in Q^{n+1}$ where $g(x)=\sum_{i=0}^{n+1} g_{i} x^{i}$, with $p \nmid g_{1}$ and $p^{m-1} \mid g_{i}$ for $2 \leq i \leq n$. We have

$$
h g(x)=\sum_{i=0}^{n} h_{i}\left(\sum_{j=0}^{n+1} g_{j} x^{j}\right)^{i}=h_{0}+h_{1} \sum_{i=0}^{n+1} g_{j} x^{j}+\sum_{i=2}^{n} h_{i}\left(\sum_{j=0}^{n+1} g_{j} x^{j}\right)^{i} .
$$


Since $p^{m-1} \mid h_{i}$, for $i \geq 2$ and $p^{m-1} \mid g_{j}$ for $j \geq 2$, any terms in $\sum_{i=2}^{n} h_{i}\left(\sum_{j=0}^{n+1} g_{j} x^{j}\right)^{i}$ involving $g_{j}$ for $j \geq 2$ vanish modulo $p^{m}$. Therefore we have

$$
h g(x)=h_{0}+h_{1} \sum_{j=0}^{n+1} g_{j} x^{j}+\sum_{i=2}^{n} h_{i}\left(g_{0}+g_{1} x\right)^{i} .
$$

By [5, Lemma 2.1], we have

$$
g^{-1}(x)=\sum_{i=1}^{n+1} b_{i} x^{i} \quad \text { with } b_{1}=g_{1}^{-1} \text { and } b_{i}=-g_{i} g_{1}^{-(i+1)} \text { for } 2 \leq j \leq n+1 .
$$

We now compute $g^{-1} h g$ in order to prove that it is in $Q_{1}^{n}$. This is given by

$$
\begin{aligned}
g^{-1} h g(x)= & \sum_{k=1}^{n+1} b_{k}\left(h_{0}+h_{1} \sum_{j=0}^{n+1} g_{j} x^{j}+\sum_{i=2}^{n} h_{i}\left(g_{0}+g_{1} x\right)^{i}-g_{0}\right)^{k} \\
= & b_{1}\left(h_{0}+h_{1} \sum_{j=0}^{n+1} g_{j} x^{j}+\sum_{i=2}^{n} h_{i}\left(g_{0}+g_{1} x\right)^{i}-g_{0}\right) \\
& +\sum_{k=2}^{n+1} b_{k}\left(h_{0}+h_{1} \sum_{j=0}^{n+1} g_{j} x^{j}+\sum_{i=2}^{n} h_{i}\left(g_{0}+g_{1} x\right)^{i}-g_{0}\right)^{k}
\end{aligned}
$$

As $p^{m-1} \mid g_{j}$ for $j \geq 2$, we have $p^{m-1} \mid b_{k}$ for $k \geq 2$. Furthermore, $p^{m-1} \mid h_{i}$ for $i \geq 2$, and thus

$$
\begin{aligned}
g^{-1} h g(x)= & b_{1}\left(h_{0}+h_{1} \sum_{j=0}^{n+1} g_{j} x^{j}+\sum_{j=0}^{n+1} h_{i}\left(g_{0}+g_{1} x\right)^{i}-g_{0}\right) \\
& +\sum_{k=2}^{n+1} b_{k}\left(h_{0}+h_{1}\left(g_{0}+g_{1} x\right)-g_{0}\right)^{k} .
\end{aligned}
$$

Let $g^{-1} h g(x)=\sum_{m=0}^{n+1} c_{m} x^{m}$, and note that $c_{n+1}=b_{1} h_{1} g_{n+1}+b_{n+1}\left(h_{1} g_{1}\right) n+1$. Then by replacing the $b_{i}$ with their values from (2), we obtain

$$
c_{n+1}=g_{1}^{-1} h_{1} g_{n+1}-g_{n+1} g_{1}^{-(n+2)} h_{1}^{n+1} g_{1}^{n+1}=g_{1}^{-1} h_{1}\left(g_{n+1}-g_{n+1} h_{1}^{n}\right) .
$$

As $h_{1} \equiv 1 \bmod p^{m-1}$, we have $h_{1}^{n} \equiv 1 \bmod p^{m-1}$. In addition, since $p^{m-1} \mid g_{n+1}$, we have $g_{n+1} h_{1}^{n} \equiv g_{n+1} \bmod p^{m}$. Therefore, $c_{n+1}=0$, and also $p^{m-1} \mid c_{i}$ for $2 \leq i \leq n$. Then we only need to show that $c_{1} \equiv 1 \bmod p^{m-1}$. Since $g_{j} \equiv 0 \bmod p^{m-1}$ for $j \geq 2, h_{i} \equiv 0 \bmod p^{m-1}$ for $i \geq 2$, and $b_{k} \equiv 0 \bmod p^{m-1}$ for $k \geq 2$, then $c_{1} \equiv$ $b_{1} \bar{h}_{1} g_{1} \bmod p^{m-1}$. Finally, as $b_{1}=g_{1}^{-1}$, we have $c_{1} \equiv h_{1} \equiv 1 \bmod p^{m-1}$.

Lemma 9 Let $1 \leq n<p-1$. If $P$ is a $p$-subgroup of $S_{p^{m}}$ with $Q_{1}^{n} \leq P \leq Q^{n+1}$, then $P=Q_{1}^{n+1}$. 
Proof By Lemma 8 Part (ii), we have $Q_{1}^{n} \triangleleft Q^{n+1}$. Hence we can consider $\bar{Q}=$ $Q^{n+1} / Q_{1}^{n}$, which is of order $p^{m-1}(p-1)$ by Lemma 8 . Let $N$ be the number of Sylow $p$-subgroups of $\bar{Q}$. Then by Sylow's Theorem, $N \equiv 1 \bmod p$ and $N$ divides $p^{m-1}(p-1)$. Hence $N=1$, so there exists a unique Sylow $p$-subgroup $\overline{P^{\prime}}$ of $\bar{Q}$ which is normal. From the condition on $P$ above, the image $\bar{P}$ of $P$ in $\bar{Q}$ is also a Sylow $p$-subgroup of $\bar{Q}$. Since there is a unique Sylow $p$-subgroup $\overline{P^{\prime}}=\bar{P}$, by Lemma 8 the image $\bar{Q}_{1}^{n+1}$ of $Q_{1}^{n+1}$ in $\bar{Q}$ is a Sylow $p$-subgroup of $\bar{Q}$. Hence $\bar{Q}_{1}^{n+1}=$ $\bar{P}=\overline{P^{\prime}}$. As $Q_{1}^{n} \lesseqgtr P$ and $Q_{1}^{n} \leq Q_{1}^{n+1}$, the result follows.

Theorem 10 The group $Q_{1}^{1}$ is a normal subgroup of $Q^{1}$ and is the unique subgroup of $S_{p^{m}}$ of order $p^{m+1}$ which contains $T$.

Proof It is obvious that $T \in Q_{1}^{1}$. By Lemma $8,\left|Q_{1}^{1}\right|=p^{m+1}$. Consider now an element $g$ of $Q^{1}, g(x)=b_{0}+b_{1} x$ with $b_{0}, b_{1} \in \mathbb{Z}_{p^{m}}$ and $\left(b_{1}, p\right)=1$. It is not difficult to check that the inverse of $g$ in $Q^{1}$ is given by $g^{-1}(x)=-b_{1}^{-1} b_{0}+b_{1}^{-1} x$. Consider $f \in Q_{1}^{1}$, so that $f(x)=a_{0}+a_{1} x$ with $a_{0}, a_{1} \in \mathbb{Z}_{p^{m}},\left(a_{1}, p\right)=1$ and $a_{1} \equiv 1 \bmod p^{m}$. We then have $g^{-1} f g(x)=g^{-1}\left(a_{0}+a_{1}\left(b_{0}+b_{1} x\right)\right)=\left(-b_{0}+a_{0}+a_{1} b_{0}\right) b_{1}^{-1}+a_{1} x$. This proves that $g^{-1} f g(x) \in Q_{1}^{1}$. Hence $Q_{1}^{1}$ is normal in $Q^{1}$. Now let $S$ be a subgroup of $Q^{1}$ of order $p^{m+1}$ which contains $T$. Thus $\langle T\rangle$ has index $p$ in $S$, and thus $\langle T\rangle$ is maximal in $S$. Furthermore, $\langle T\rangle \triangleleft S$, because any subgroup of a $p$-group of index $p$ must be normal. Therefore we have $S=N_{S}(T) \leq N_{S_{p^{m}}}(T)$, and by Lemma 8, $S \leq N_{S_{p^{m}}}(T)=A G\left(p^{m}\right)=Q^{1}$. Thus, such an $S$ must be a subgroup of $Q^{1}$. It is clear that $Q_{1}^{1}$ is not abelian, and $S$ cannot be abelian since it is a transitive group. If this were the case it would have to be a regular group [23, Theorem 1.6.3], and thus $|S|=p^{m}$, which is impossible. Furthermore, the $p$-groups which contain a cyclic maximal subgroup are known [23, Theorem 5.3.4]. If these groups are not abelian or $p \neq 2$, they have the following special forms:

$$
Q_{1}^{1}=\left\langle x, T \mid x^{p}=1 ; x^{-1} T x=T^{1+p^{m-1}}\right\rangle,
$$

and

$$
S=\left\langle y, T \mid y^{p}=1 ; y^{-1} T y=T^{1+p^{m-1}}\right\rangle .
$$

However, the conditions on $x$ and $y$ give

$$
x^{-1} T x=y^{-1} T y \quad \Longleftrightarrow T y x^{-1}=y x^{-1} T,
$$

so the only elements of $S_{p^{m}}$ which commute with $T$ (a complete cycle of length $p^{m}$ ), are the powers of $T$. Thus $y x^{-1}=T^{j}$ for some $j$. Since the order of $y x^{-1}$ is $p$, the only choices for $j$ are $j=p^{m}$ or $j=p^{m-1}$. For both choices we get $S=Q_{1}^{1}$, namely $j=p^{m}$ gives $x=y^{-1}$ (so $S=Q_{1}^{1}$ ), and $j=p^{m-1}$ gives $x=T^{-p^{m-1}} y$. Thus we have $x \in\langle y, T\rangle$, so that $\langle x, T\rangle=\langle y, T\rangle$, and hence $S=Q_{1}^{1}$.

Theorem 11 Let $p$ be an odd prime, $q=r^{\alpha}$ a prime power, $C$ a cyclic code over $\mathbb{F}_{q}$ of length $p^{m}$, and $P$ a Sylow $p$-subgroup of Per $(C)$ of order $p^{s}$ such that $T \in P$. Then the following hold: 
(a) If $p \nmid \alpha$ and $p \nmid\left(d, r^{a}-1\right)$, then $s=m$, and $P=\langle T\rangle$ if and only if $\operatorname{Per}(C)=$ $P \Gamma L\left(d, r^{a}\right), d \geq 3$

(b) If $p \geq 5, \alpha=1$ and $r=p, m>1$, then $\operatorname{Per}(C)$ is an imprimitve group and $P$ is normal of order $p^{s}, s>m$. If $m<s \leq p+m-1$, then we have $P=Q_{1}^{s-m}$.

(c) If $z=1, p \nmid \alpha$ and $p \nmid\left(d, r^{a}-1\right)$, then $\operatorname{Per}(C)$ is an imprimitve group and $P$ is normal of order $p^{s} \geq p^{2 m-1}$. Furthermore, if $2 m-1<s \leq p+m-1$, then we have $P=Q_{1}^{s-m}$.

Proof Statement (a) and the first parts of (b) and (c) follow from Theorem 7. We thus only need prove that if $s<p+m-1$, then $P=Q_{1}^{s-m}$. Assume $s \leq p+m-1$, so that $P$ contains a $p$-subgroup $P^{\prime}$ of order $p^{m+1}$. By Theorem 10 , we obtain $P^{\prime}=Q_{1}^{1}$. Let $j \geq 1$ be the largest integer such that $Q_{1}^{j} \leq P$. If $j=p-1$, by Lemma 8 we have $\left|Q_{1}^{p-1}\right|=p^{p+m-1}$. Thus $Q_{1}^{p-1}$ is a subgroup of $P$ of the same order as $P$, and hence $P=Q_{1}^{p-1}$, so we can assume that $1 \leq j<p-1$. If $Q_{1}^{j} \lesseqgtr P$, then $Q_{1}^{j} \lesseqgtr N_{P}\left(Q_{1}^{j}\right)$ and by Lemma $8, N_{P}\left(Q_{1}^{j}\right) \leq Q_{1}^{j+1}$. Since $Q_{1}^{j} \lessgtr N_{P}\left(Q_{1}^{j}\right) \leq Q_{1}^{j+1}$, by Lemma 8 $N_{p}\left(Q_{1}^{j}\right)=Q_{1}^{j+1}$, which contradicts the choice of $j$.

\section{References}

1. Bardoe, M., Sin, P.: The permutation modules for $G L\left(n+1, \mathbb{F}_{q}\right)$ acting on $\mathbb{P}^{n}\left(\mathbb{F}_{q}\right)$ and $\mathbb{F}_{q}^{n+1}$. J. Lond. Math. Soc. 61, 58-80 (2000)

2. Berger, T.P.: A direct proof for the automorphism group of Reed-Solomon codes. In: Cohen, G., Charpin, P. (eds.) Proc. Eurocode 90. Lecture Notes in Computer Science, vol. 514, pp. 21-29. Springer, Berlin (1991)

3. Berger, T.P., Charpin, P.: The permutation group of affine invariant extended cyclic codes. IEEE Trans. Inf. Theory 62(6), 2194-2209 (1996)

4. Bienert, R., Klopsch, B.: Automorphism group of cyclic codes. J. Algebr. Comb. 31, 33-52 (2010)

5. Brand, N.: Polynomial isomorphisms of combinatorial objects. Graphs Comb. 7(1), 7-14 (1991)

6. Brillhart, J., Tonascia, J., Weinberger, P.: On the Fermat quotient. In: Atkin, A.O.L., Birch, B. (eds.) Computers in Number Theory. Academic Press, New York (1991)

7. Burnside, W.: On some properties of groups of odd order. J. Lond. Math. Soc. 33, 162-185 (1901)

8. Burnside, W.: Theory of Groups of Finite Order. Dover, Mineola (1955)

9. Cameron, P.J.: Finite permutation groups and finite simple groups. Bull. Lond. Math. Soc. 13, 1-22 (1981)

10. Demazure, M.: Cours D’Algèbre: Primalité, Divisibilité, Codes. Cassini, Paris (1997)

11. Dixon, J.D., Mortimer, B.: Permutation Groups. Graduate Texts in Mathematics, vol. 163. Springer, Berlin (1996)

12. Dixon, J.D., Zalesskii, A.: Finite primitive linear groups of prime degree. J. Lond. Math. Soc. 57(2), 126-134 (1998)

13. Dobson, E.D.: On groups of odd prime-power degree that contain a full cycle. Discrete Math. 299, 65-78 (2005)

14. Dobson, E.D., Witte, D.: Transitive permutation groups of prime-squared degree. J. Algebr. Comb. 16(1), 43-69 (2002)

15. Huffman, W.C., Job, V., Pless, V.: Multiplier and generalized multipliers of cyclic objects and cyclic codes. J. Comb. Theory, Ser. A 62, 183-215 (1993)

16. Huffman, W.C.: Codes and groups. In: Pless, V.S., Huffman, W.C. (eds.) Handbook of Coding Theory, vol. II, pp. 1345-1439. Elsevier, Amsterdam (1998)

17. Huppert, B., Blackburn, N.: Finite Groups II. Grundlehren Math. Wiss., vol. 242. Springer, Berlin (1982)

18. Lim, F., Fossorier, M., Kavcić, A.: Notes on the automorphism group of Reed-Solomon binary images. In: Proc. IEEE Int. Symp. Inform. Theory, Toronto, Canada, July 2008, pp. 1813-1817 (2008) 
19. MacWilliams, F.J., Sloane, N.J.A.: The Theory of Error Correcting-Codes. North-Holland, Amsterdam (1977)

20. McEliece, R.J.: A public-key cryptosystem based on algebraic coding theory. DSN Progress Report 42-44, pp. 114-116, Jan.-Feb. 1978

21. McSorley, J.P.: Cyclic permutation groups in doubly-transitive groups. Commun. Algebra 25, 33-35 (1997)

22. Mortimer, B.: The modular permutation representations of the known doubly transitive groups. Proc. Lond. Math. Soc. 41, 1-20 (1980)

23. Robinson, D.J.S.: A Course in the Theory of Groups. Graduate Texts in Mathematics, vol. 80. Springer, Berlin (1980)

24. Roth, R., Seroussi, G.: On cyclic MDS codes of length $q$ over $G F(q)$. IEEE Trans. Inf. Theory 32(2), 284-285 (1986)

25. Wielandt, H.: Finite Permutation Groups. Academic Press, New York (1964) 